\title{
Enhanced inactivated poliovirus vaccine
}

$\mathrm{B}$ OTH INACTIVATED (IPV) AND LIVE, ORAL (OPV) POLIOVIRUS vaccine are licensed for use in Canada. IPV consists of the three wild poliovirus serotypes grown on human diploid cells (MRC-5) and inactivated with formalin. The IPV now available is termed enhanced IPV (eIPV) because of a new method of production developed in 1978 that results in higher potency per dose and significantly greater immunogenicity than the original IPV (1). Both separate IPV and combination products containing diphtheria-pertussis-tetanus (DPT)-IPV, diphtheria-tetanus-IPV and tetanus-diphtheria toxoid-polio are available. The DPT-IPV has recently been licensed for use as the diluent for haemophilus $\mathrm{b}$ conjugate vaccine (tetanus protein conjugate) (PRP-T, ActHIB) making possible a single injection of pentavalent vaccine (PENTA).

When administered alone, IPV can be injected subcutaneously. Combination vaccines containing DPT must be administered intramuscularly because of presence of alum as an adjuvant. Because thimerosal has a detrimental effect on IPV, the preservative in all vaccines containing IPV is $0.5 \% 2$-phenoxyethanol. IPV also contains $0.5 \%$ human albumin as a stabilizer, less than 1 part/million of bovine serum, trace amounts of streptomycin and neomycin, 20 parts/million of Tween 80, and 27 parts/million of formaldehyde.

IPV produces immunity to all three poliovirus serotypes. Because of greater antigen content, 99 to $100 \%$ of infants seroconvert to all three serotypes after two doses at two and four months of age (2). After three doses of eIPV or OPV at two, four and 18 months, the seroconversion rates and geometric mean serum antibody titres were significantly higher in recipients of IPV than in recipients of opv. The proportions of children with neutralizing antibody and immunoglobulin A antibody titres in nasopharyngeal secretions were similar in both vaccine groups (3). Antibodies persist for at least 10 years, but immunity memory, ie, the ability to mount a rapid anamnestic response when challenged by vaccine or infection, lasts many more years.

The risk of paralytic poliomyelitis associated with the use of OPV coupled with the availability of the new pentavalent vaccine for infant immunization containing DPT, IPV and haemophilus b conjugate vaccine is leading many provinces in Canada to consider changing from OPV to IPV. The success of the polio eradication program in the western hemisphere means that the risk of disease due to wild-type poliovirus is now less than the risk of vaccine-associated disease in Canada. Although polio is still present in many parts of the world, effective immunization of the Canadian population will minimize the risk of spread of imported polioviruses.

The optimal polio immunization schedule has yet to be determined. It is not clear whether a sequential IPV-OPV schedule will be superior to IPV alone. If the World Health Organization polio eradication program proves to be as successful in the rest of the world as it has been in Central and South America, the need for opv may disappear.

\section{REFERENCES}

1. Bernier R. Improved inactivated poliovirus vaccine: an update. Pediatr Infect Dis J 1986;5:289-92.

2. Faden H, Modlin J, Thoms M, McBean A, Ferdon M, Ogra P. Comparative evaluation of immunization with live attenuated and enhanced-potency inactivated trivalent poliovirus vaccines in childhood: systemic and local immune responses. J Infect Dis 1990;162:1291-7.

3. McBean A, Thoms M, Albrecht P, et al. Serologic response to oral polio vaccine and enhanced-potency inactivated polio vaccines. Am J Epidemiol 1988; 128:615-28.
Ronald Gold MD Division of Infectious Disease The Hospital for Sick Children Toronto, Ontario

All material presented in Pediatric Infectious Disease Notes has been reviewed and approved by the chairperson, Canadian Paediatric Society Board and representative members of the Canadian Paediatric Society Committee on Infectious Diseases and Immunization

Correspondence: Infectious Diseases and Immunization Committee, Canadian Paediatric Society. 401 Smyth Rd, Ottawa, Ontario K1H 8L1. Telephone (613) 737-2728, Fax (613) 737-2794 


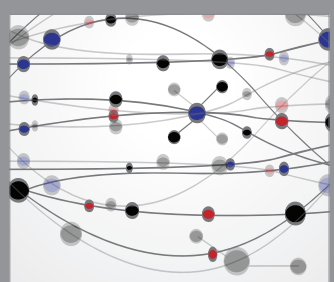

The Scientific World Journal
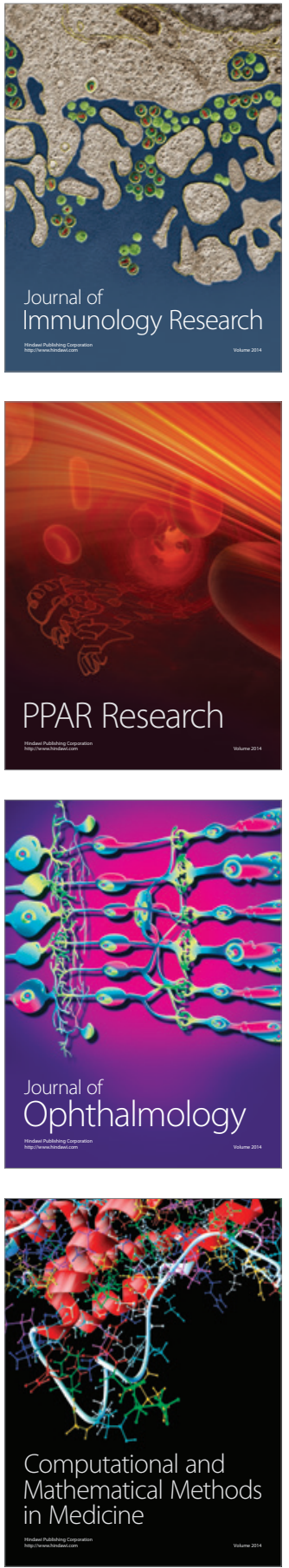

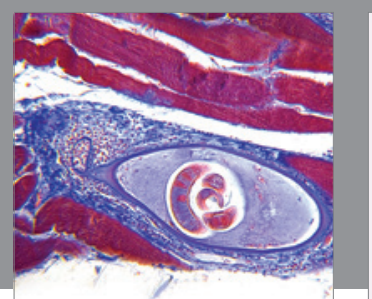

Gastroenterology Research and Practice

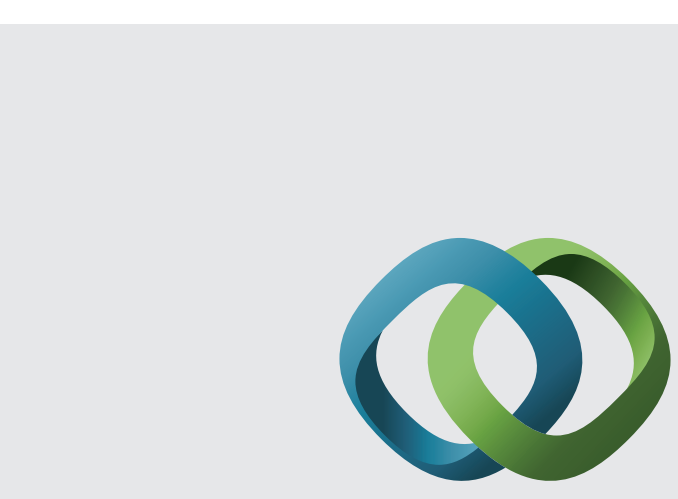

\section{Hindawi}

Submit your manuscripts at

http://www.hindawi.com
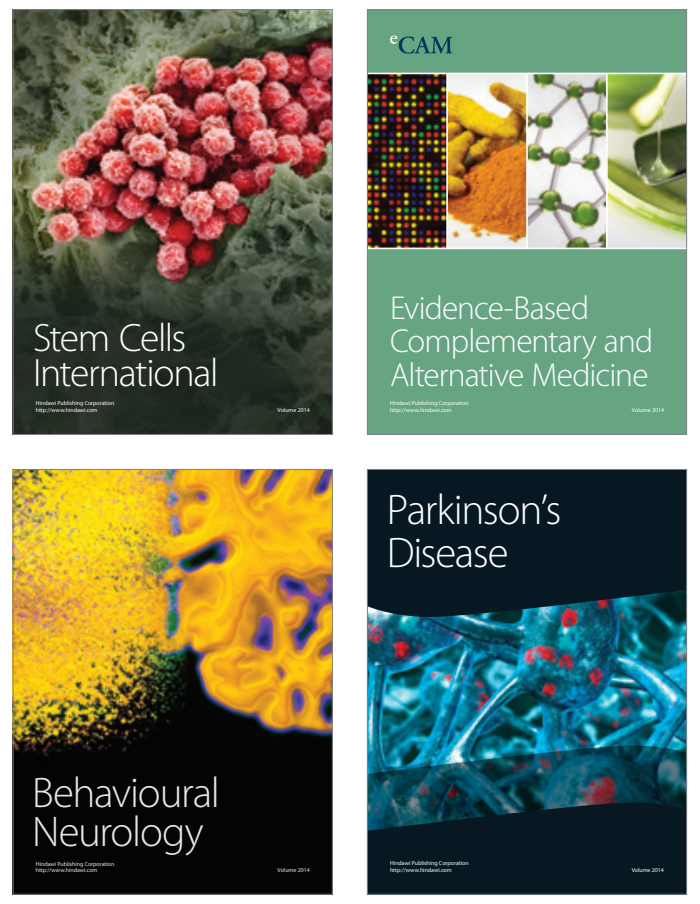
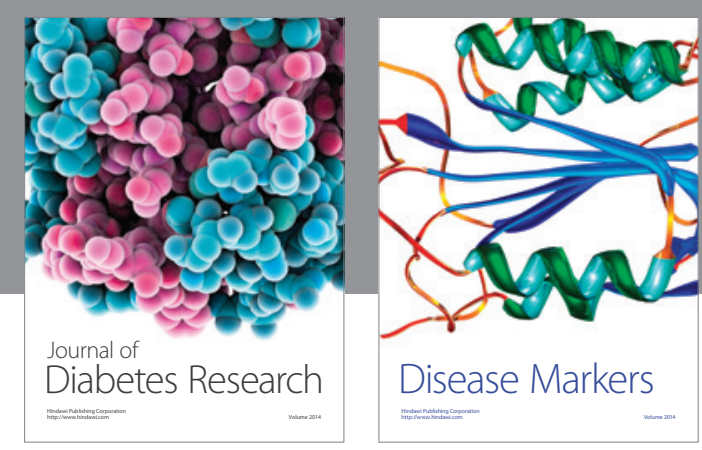

Disease Markers
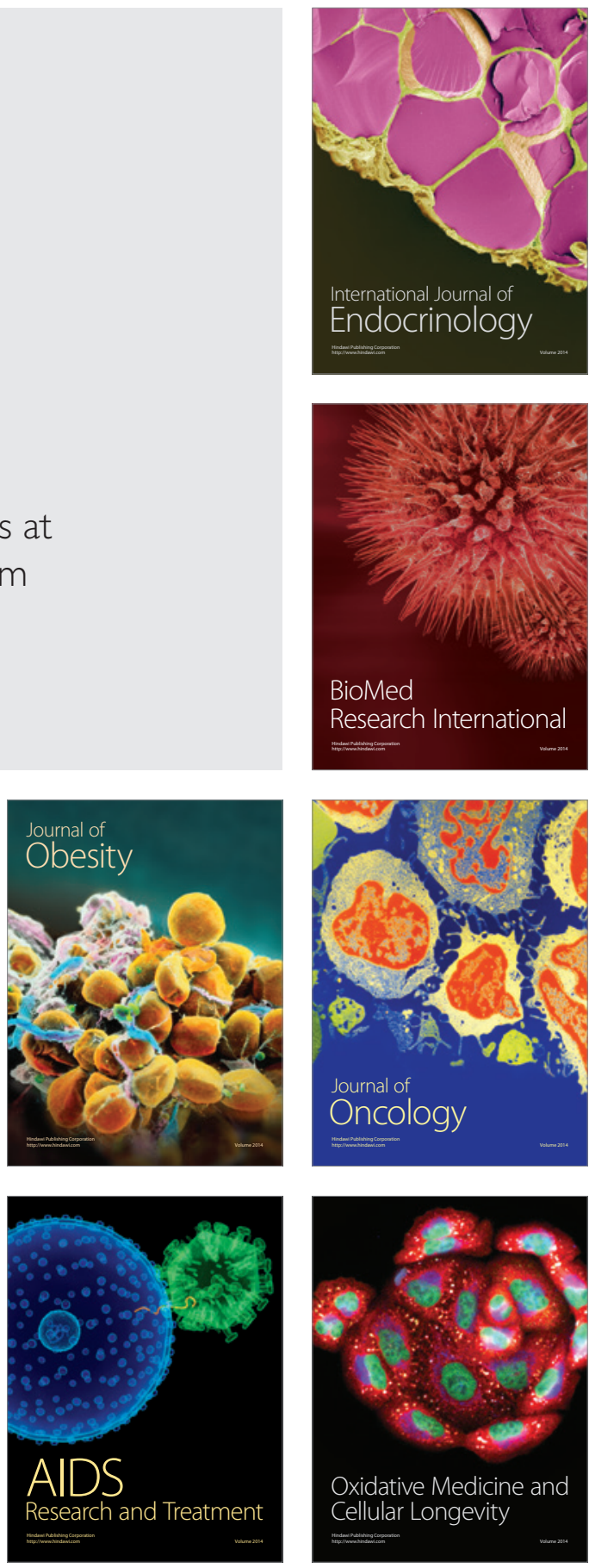